\title{
Two- and Three-Dimensional Flexible Grids for Reservoir Simulation
}

\author{
VERMA, Santosh, Stanford University, \\ AZIZ, Khalid, Stanford University
}

Paper presented at the 5th European Conference on the Mathematics of Oil Recovery, Leoben, Austria, 3-6 Sept. 1996

\begin{abstract}
The commonly used Cartesian and hybrid local grid refinements have the disadvantage that the base grid is always Cartesian, which makes it difficult to align along reservoir heterogeneities. Also, the locally orthogonal Voronoi and $k$-orthogonal perpendicular bisector (PEBI) grids are only useful for isotropic or limited anisotropic permeability distributions. The finite-difference approach proposed in this paper overcomes limitations of existing flexible gridding schemes in modeling full, anisotropic and asymmetric permeability tensors and permeability heterogeneity. The new scheme assumes uniform properties inside control-volumes and it can be used for control volumes formed around vertices of triangles in two-dimensions and tetrahedra in threedimensions. It can thus be used with Voronoi grids in twodimensions, and median (i.e. CVFE) and boundary adapting grids (BAG) in two- and three-dimensions. Applications of Voronoi grids in three-dimensions are limited due to geometrical considerations. The proposed method can also be used with grids constructed to align along major reservoir heterogeneities, wells and streamlines. Several applications of this method for two- and three-dimensional problems are presented.
\end{abstract}

\section{INTRODUCTION}

Petroleum reservoir simulation involves the numerical solution of mass and/or energy conservation equations in discretized form over a grid. A grid is called flexible (or unstructured) when it is made up of polygons (polyhedra in three-dimensions) whose shape and size can vary from place to place in the reservoir. This paper addresses two important aspects of flexible grids; (a) grid construction, and (b) numerical procedure to solve fluid flow equations on these grids.

Solution of fluid and heat flow equations on flexible grids have been discussed extensively in the literature (e.g. Heinrich 1987, Heinemann 1988, Palagi 1992 and Prakash 1987). Most of the existing numerical schemes assume either an isotropic permeability tensor or a symmetric and anisotropic permeability tensor. Existing methods do not provide sufficient flexibility to align grids with (a) reservoir bed boundaries, (b) beds with varying permeability ten- sors, (c) faults and (d) horizontal/inclined wells. The new method proposed in this work provides greater flexibility than existing approaches.

\section{PREVIOUS WORK}

In this section, some of the most important previous works related to the topic of this paper are described.

Local Grid Refinement (LGR). Local grid refinement involves using fine grid inside coarse base grid. This is done only in selected regions, e.g., near-well regions, in regions of wide saturation variation, in highly heterogeneous regions, etc. This reduces computation time compared to uniform fine grids. The two commonly used methods are Cartesian local grid refinement (CLGR) (Ciment and Sweet 1973 and Nacul 1991) as shown in Figure 1 and hybrid local grid refinement (HLGR) as shown in Figure 2 (Pedrosa and Aziz, 1985).

Both the Cartesian and hybrid LGR require a base grid which is Cartesian. Hence it becomes difficult to align the grid with varying facies, horizontal wellbores, etc. Also, additional discretization errors are introduced at the boundaries of coarse and refined regions.

Control Volume Finite Element (CVFE) Method. The control volume finite element (CVFE) method uses triangular mesh in two-dimensions (Figure 3) and tetrahedral mesh in three-dimensions. Control volumes are formed around gridnodes by joining the midpoint of the triangles' edges with a point $\alpha$ inside the triangle. Different locations of point $\alpha$ give rise to different forms of the flow term between grid nodes. When $\alpha$ is at the barycenter, the resulting grid is called CVFE type grid (Heinrich, 1987, calls this the median grid. Forsyth (1989) applied the CVFE method to thermal reservoir simulation problems. However, all his numerical examples were for homogeneous reservoirs.

Fung et al. (1991) also studied the CVFE method and implemented it in a general purpose thermal simulator. They converted the algebraic approximation of the conservation equation to a form similar to those in conventional finite-difference simulators, thus making its implementation in existing reservoir simulators easy. They showed 
that grid orientation errors can be substantially reduced by choosing proper triangular elements. To simulate threedimensional flow they did a vertical projection of the two-dimensional grid. Fung et al. (1993) also presented a hybrid-CVFE method to improve the representation of wells. They used cylindrical or elliptical grid near well regions and the CVFE grid in rest of the reservoir. This scheme assumes the permeability tensor inside each triangular element to be uniform. As shown by Verma (1995, 1996a) this may lead to significant numerical errors when permeability contrast between the triangles is large. Sonier and Eymard (1993) presented some mathematical and numerical properties of CVFE method.

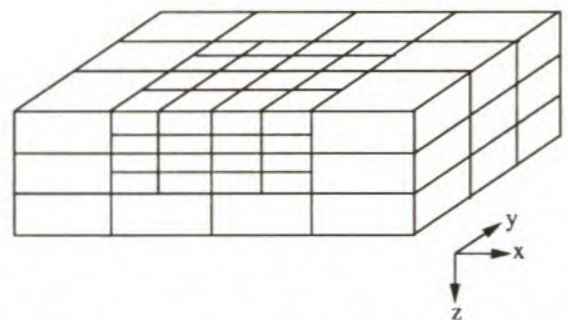

Figure 1: Hybrid local grid refinement

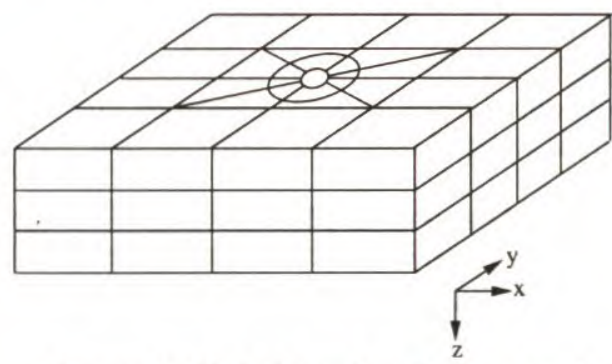

Figure 2: Hybrid local grid refinement

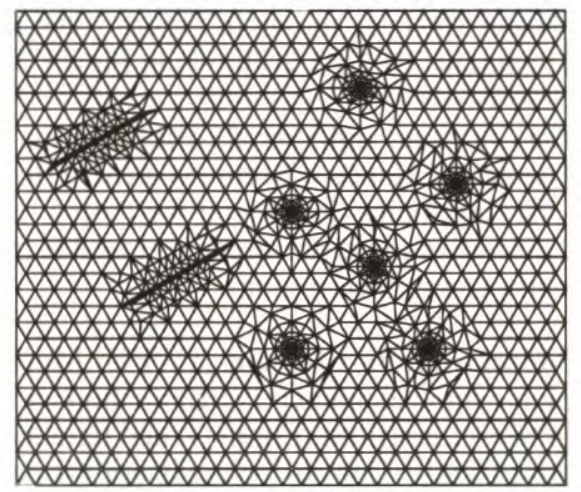

Figure 3: A CVFE mesh of connections

CVFD Method: Voronoi (PEBI) and GPEBI Grid. The method of perpendicular bisectors (PEBI) was introduced by Heinrich (1987). The PEBI grid (also called Voronoi grid) is locally orthogonal, i.e., the block boundaries are normal to lines joining the nodes on the two sides of each boundary (Figure 4). This allows a reasonably accurate computation of interblock transmissibility for heterogeneous but isotropic permeability distribution. Heinemann and Brand (1988, 1989) and Heinemann (1994) describe practical methods for using PEBI grids to represent reservoir geometry.

Heinemann and Brand $(1988,1989)$ also presented a

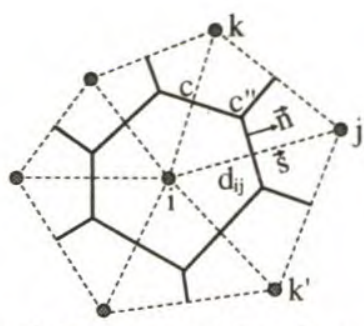

Figure 4: Voronoi gridblock example

method of implementing the PEBI method for full and anisotropic but symmetric permeability tensors. The grid block boundaries in this case are not locally orthogonal but oriented in a manner such that the vector $\overline{\bar{k}} \vec{n}$, (where $\overline{\bar{k}}$ is the permeability tensor in the triangle and $\vec{n}$ is the outward normal to the block surface) is parallel to $\vec{s}$, the vector pointing to node $j$ from node $i$. A result of this simplification is that flow across any surface becomes only a function of potentials at the nodes on either side of that surface. Such grids have been called $k$-orthogonal or generalized perpendicular bisector (GPEBI) grids (Heinemann and Brand, 1988). Figure 5 shows a GPEBI gridblock. This approach however runs into problems for regions having high permeability anisotropy (Verma, 1995 and 1996a).

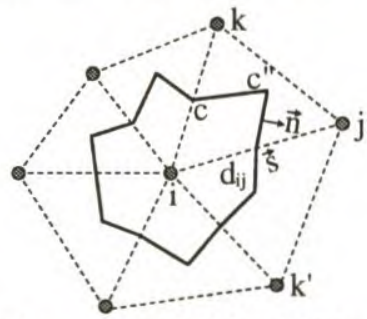

Figure 5: Generalized PEBI gridblock example

Palagi and Aziz (1991) and Palagi (1992) have also studied Voronoi (PEBI) grids to model reservoir geometry. They describe a practical way of using Voronoi grids for field scale simulation by taking advantage of pre-defined geometrical modules which can be located, scaled and rotated in any position. Figure 6 is an example of a grid generated using several modules. They used the control volume finite difference (CVFD) method on this grid. The CVFD method, just like the traditional 5-point method on Cartesian grids, computes the velocity at the gridblock boundary based only on the potentials at the two nodes on either side of the gridblock boundary. This method is restricted to isotropic permeability.

Results presented by both Heinemann and Brand (1989) and Palagi and Aziz (1991) show major applications of flexible grid in two-dimensions only. Layers of such grids are stacked to represent the variation in the third dimension. Aziz (1993) reviewed gridding methods and suggested guidelines for grid selection.

Flux Continuous Scheme. Edwards and Rogers (1994) presented a flux continuous scheme for the full tensor pressure equation to model single phase fluid flow with quadrilateral grids in two-dimensions. These grids are nonorthogonal and use flux balances across the quadrilateral block boundaries by constraining the $x$ and $y$ direction fluxes. Also, potentials are constrained at specific points on the boundaries. Such potential and flux constraints are used to obtain transmissibility coefficients which are similar to the harmonic average transmissibility used in 


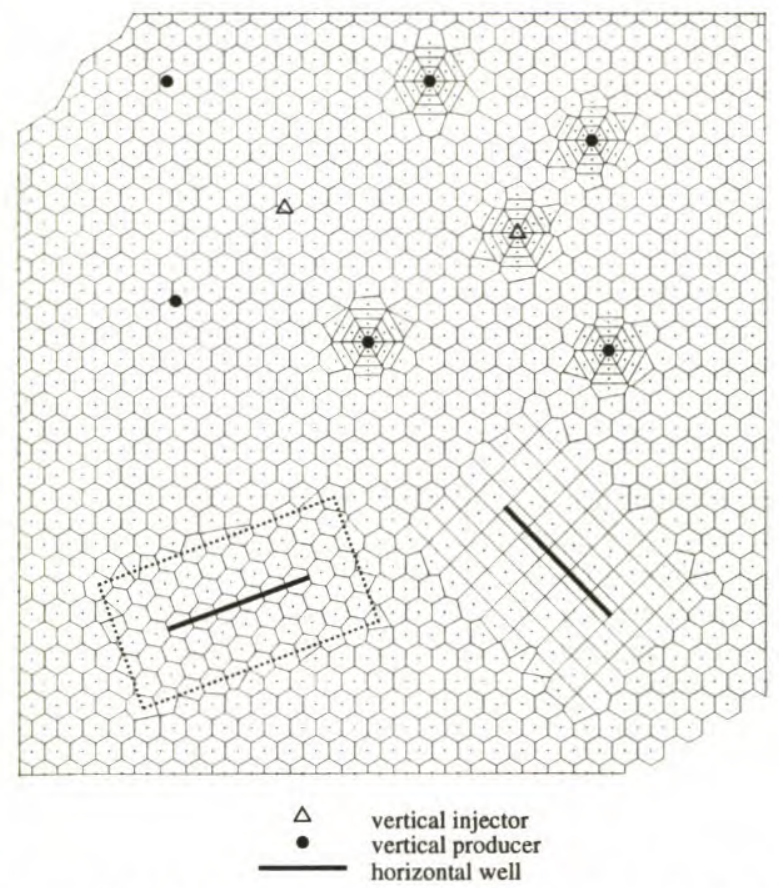

Figure 6: A Voronoi grid constructed with several modules (after Palagi and Aziz, 1991)

reservoir simulation. Verma (1995) presented numerical schemes for Voronoi grids having uniform properties inside the gridblock. This scheme uses flux constraints normal to the grid block boundaries.

Aavatsmark et al. (1994a) presented a method for discretization on nonorthogonal, curvilinear grids for multiphase flow. Aavatsmark et al. (1995a) presented a scheme similar to that of Edwards and Rogers for discretization on nonorthogonal quadrilateral grids for inhomogeneous and anisotropic media while Aavatsmark et al. (1995b) presented a discretization method on triangular grids for general media. This scheme was developed in parallel and independently of the work discussed here.

\section{MATHEMATICAL AND NUMERICAL MODEL}

The mathematical model used for fluid flow simulation in petroleum reservoirs is discussed in this section. Flow equation for any component is formulated by combining mass conservation with Darcy's law.

Mass Conservation Equation. Eq. 1 represents the law of mass conservation for an arbitrary component $c$ in a control volume $V$ with an external area $A$, assuming that diffusion and dispersion can be neglected and that there are no chemical reactions (Lake, 1989):

$$
-\oint_{A} \sum_{p=1}^{n_{p}} \omega_{c p} \vec{v}_{p} \cdot \vec{n} d A=\frac{\partial}{\partial t} \int_{V} \sum_{p=1}^{n_{p}} \omega_{c p} S_{p} \phi d V
$$

Here subscript $p$ denotes phase, subscript $c$ denotes component and $n_{p}$ is the number of phases. One such equation is written for each component $c$ in the system. The flow term, which is on the left hand side (LHS), can have terms due to (a) flow across surfaces which are inside the reservoir, (b) flow across surfaces which are at the boundary of the reservoir, and/or (c) flow through wells completed in the gridblock. The summation over $n_{p}$ in the flow and accumulation terms represents the fact that component $c$ can be present in any of the $p$ phases. Concentration $\omega_{c p}$ is the product of $x_{c p}$, mass fraction of component $c$, in phase $p$, and $\rho_{p}$, the density of phase $p . \vec{v}_{p}$ is the Darcy velocity of phase $p, \vec{n}$ is the outward normal to the surface (area $A$ ) of the control volume, $\phi$ is the porosity of the medium, and $S_{p}$ is the saturation of phase $p$. The discretizations of Eq. 1 presented in this paper are valid for multicomponent and multiphase flow, but for simplicity the discussion presented here is for two immiscible phases: oil and water. For this case $x_{c p}=1$, hence $\omega_{c p}=\rho_{p}$, which can be written as

$$
\rho_{p}=\frac{\bar{\rho}_{p}}{B_{p}}
$$

where $\bar{\rho}_{p}$ is density of phase $p$ at standard conditions and $B_{p}$ is its formation volume factor. The velocity vector $\vec{v}_{p}$ is given by

$$
\vec{v}_{p}=-\frac{k_{r p}}{\mu_{p}} \overline{\bar{k}} \vec{\nabla} \Phi_{p}
$$

where $k_{r p}$ is the relative permeability of phase $p, \mu_{p}$ is its viscosity, $\overline{\bar{k}}$ is the permeability tensor, and $\vec{\nabla} \Phi_{p}$ is the potential gradient in phase $p$. Putting the expression for $\vec{v}_{p}$ and $\omega_{c p}$ in Eq. 1 and assuming that water and oil components can exist only in their respective phases the following equation is obtained for $p=o, w$ :

$$
\oint_{A} \frac{k_{r p}}{\mu_{p} B_{p}} \overline{\bar{k}} \vec{\nabla} \Phi_{p} \cdot \vec{n} d A=\frac{\partial}{\partial t} \int_{V} \frac{S_{p} \phi}{B_{p}} d V .
$$

Proposed Numerical Method. In this section a new discretization scheme for equation Eq. 4 is described and expressions for flux across the control volume surfaces are derived. The scheme is initially presented for grids which obey the Voronoi criterion with the limitation that the circumcenter of each triangle/tetrahedron be inside the triangle/tetrahedron. Deviations from this limitation will be discussed. This discretization scheme can also be used for CVFE type grid.

Figure 7 shows a tetrahedron and parts of Voronoi blocks around each gridnode. Solution of the unknowns is sought at gridnodes $0,1,2$ and 3 . In the CVFE and GPEBI discretization it is assumed that the properties are uniform inside each triangular (tetrahedral in three-dimensions) region. Because of this assumption it is very easy to derive an expression for the pressure gradient at the interfaces and hence flow across interfaces. But, in the current formulation, permeability varies inside a tetrahedron, but is constant inside the control volume. Figure 7 shows four permeability regions in the tetrahedron; one associated with each vertex. Hence no straightforward linear combination of potentials at the vertices can be used to estimate potential gradient inside the tetrahedral region. Regardless of the assumptions made in arriving at an expression for the potential gradient, flux continuity must be maintained across grid block faces inside each tetrahedron. Also potential continuity must be maintained at a specific point on each interface. In the case of Cartesian grids potential continuity is normally achieved at gridblock face centers and flux continuity is maintained across each gridblock face, which leads to the harmonic averaging of interblock transmissibility. 


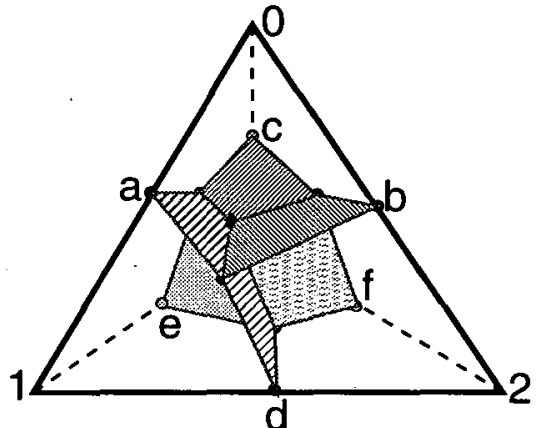

Figure 7: Constraint points and boundaries for a tetrahedron

Three-Dimensional Transmissibility for Flexible Grids. Exactly the same principles as used for determining interblock transmissibility for Cartesian grids can be used to determine transmissibility for flexible grids in three-dimensions. Figure 7 shows the decomposition of a tetrahedron into homogeneous control volumes associated with the vertices of the tetrahedron. It is assumed that porosity and permeability tensor inside each control volume are homogeneous. The permeability tensor may be full, asymmetric and anisotropic.

A tetrahedron has four homogeneous regions, one surrounding each node and there are six interfaces inside each tetrahedron. There will thus be six flow terms inside each tetrahedron. A face potential is introduced at each interface (just like in the 1D case). These face potentials along with the potentials at the nodes are used to estimate potential gradients inside each of the control volumes in the tetrahedron which can then be used to estimate velocity at each interface. These velocities are used to estimate the flux at each interface. The flux across an interface should be the same when calculated with the properties of the control volumes on either side of the interface. This condition can be satisfied by forcing the velocities normal to the interface to be the same on both sides. Single phase flow rate, for a fluid of unit viscosity $\mu_{p}$ and unit formation volume factor $B_{p}$, across interface $a$ (i.e. the interface on which $a$ lies), based on the velocity in region 0 is

$$
q_{0 a}=-\int_{a} \vec{v}_{0} \cdot \vec{n}_{a} d A=-A_{a} \vec{v}_{0} \cdot \vec{n}_{a}
$$

where $n_{a}$ is the outward normal on the interface $a$ with node 0 considered as an inside point. A positive value of $q$ signifies flow into the block while a negative value signifies flow out of the block. Single phase velocity in region $i(i=$ $0, \ldots, 3)$ is given by:

$$
\vec{v}_{i}=-\overline{\bar{k}}_{i} \vec{\nabla} \Phi_{i}, i=0, \ldots, 3
$$

To estimate the potential gradients in the above equations we assume that potential varies linearly in regions $0, \ldots, 3$ of the tetrahedron. Using a Taylor series expansion of potentials at $a, b$ and $c$ about node 0 and retaining the first order terms gives

$$
\left[\begin{array}{l}
\Phi_{x_{0}}^{\prime} \\
\Phi_{y_{0}}^{\prime} \\
\Phi_{z_{0}}^{y_{0}}
\end{array}\right]=\left[\begin{array}{lll}
A_{x 1} & A_{x 2} & A_{x 3} \\
A_{y 1} & A_{y 2} & A_{y 3} \\
A_{z 1} & A_{z 2} & A_{z 3}
\end{array}\right]\left[\begin{array}{c}
\Phi_{a}-\Phi_{0} \\
\Phi_{b}-\Phi_{0} \\
\Phi_{c}-\Phi_{0}
\end{array}\right](7)
$$

In a similar manner expressions for regions 1,2 and 3 are obtained.
Flow rates across the interfaces are based on the velocities at the points $a, b, c, d, e$ and $f$. The constraint equation at these points are:

$$
\begin{array}{ll}
\vec{v}_{0} \cdot \vec{n}_{a}=\vec{v}_{1} \cdot \vec{n}_{a}, & \vec{v}_{0} \cdot \vec{n}_{b}=\vec{v}_{2} \cdot \vec{n}_{b}, \\
\vec{v}_{0} \cdot \vec{n}_{c}=\vec{v}_{3} \cdot \vec{n}_{c}, & \vec{v}_{1} \cdot \vec{n}_{d}=\vec{v}_{2} \cdot \vec{n}_{d}, \\
\vec{v}_{1} \cdot \vec{n}_{e}=\vec{v}_{3} \cdot \vec{n}_{e}, \text { and } & \vec{v}_{2} \cdot \vec{n}_{f}=\vec{v}_{3} \cdot \vec{n}_{f}
\end{array}
$$

where $\vec{n}_{a}, \vec{n}_{b}, \vec{n}_{c}, \vec{n}_{d}, \vec{n}_{e}$, and $\vec{n}_{f}$ are outward normals. The following variables, for $u=x, y$ and $z$, and $v=a, b, c, d, e$ and $f$ are now defined:

$$
F_{u v_{i}}=k_{x u_{i}} n_{x_{v}}+k_{y u_{i}} n_{y_{v}}+k_{z u_{i}} n_{z_{v}} ; i=0,1,
$$

The expressions for the pressure gradients from Eq. 7 are put into the expressions for velocities in $\mathrm{Eq}$. 6 . The resulting expressions are put into the set of velocity constraint equations, Eq. 8. The coefficients of potentials in the velocity equations are then collected and the following matrix equation results:

$$
M R \Phi_{v}=M L \Phi_{i}
$$

where $M L$ is a $6 \times 6$ matrix and $M R$ is a $4 \times 6$ matrix and

$$
\Phi_{v}=\left[\Phi_{a} \Phi_{b} \Phi_{c} \Phi_{d} \Phi_{e} \Phi_{f}\right]^{T}
$$

and

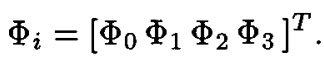

The entries of $M L$ and $M R$ matrices are given in detail by Verma (1996a).

Solving for the face potentials in terms of the node potentials using equation Eq. 10 gives:

$$
\left[\begin{array}{l}
\Phi_{a} \\
\Phi_{b} \\
\Phi_{c} \\
\Phi_{d} \\
\Phi_{e} \\
\Phi_{f}
\end{array}\right]=\left[\begin{array}{llll}
R_{00} & R_{01} & R_{02} & R_{03} \\
R_{10} & R_{11} & R_{12} & R_{13} \\
R_{20} & R_{21} & R_{22} & R_{23} \\
R_{30} & R_{31} & R_{32} & R_{33} \\
R_{40} & R_{41} & R_{42} & R_{43} \\
R_{50} & R_{51} & R_{52} & R_{53}
\end{array}\right]\left[\begin{array}{c}
\Phi_{0} \\
\Phi_{1} \\
\Phi_{2} \\
\Phi_{3}
\end{array}\right]
$$

where the $\mathrm{R}$ matrix is obtained numerically. The flow across interface $a$ at point $a$ in region 0 is given by:

$$
\begin{aligned}
q_{0 a}= & \left(k_{x x_{0}} \Phi_{x 0}^{\prime}+k_{x y_{0}} \Phi_{y 0}^{\prime}+k_{x z_{0}} \Phi_{z 0}^{\prime}\right) n_{x_{a}} \\
& \left(k_{y x_{0}} \Phi_{x 0}^{\prime}+k_{y y_{0}} \Phi_{y 0}^{\prime}+k_{y z_{0}} \Phi_{z 0}^{\prime}\right) n_{y_{a}}+ \\
& \left(k_{z x_{0}} \Phi_{x 0}^{\prime}+k_{z y_{0}} \Phi_{y 0}^{\prime}+k_{z z_{0}} \Phi_{z 0}^{\prime}\right) n_{z_{a}}
\end{aligned}
$$

The potential gradients as obtained from Eq. 7, with the values of potentials at $a, b, c, d$, etc. obtained from Eq. 11, can be used to express $q_{a}$, flux per unit area at $a$, in the form

$$
q_{a}=\sum_{i=0}^{3} T_{a i} \Phi_{i}
$$

where

$$
\begin{aligned}
T_{a 0}= & \left(R_{00}-1\right) \sum_{u} A_{u 1} F_{u a_{0}}+ \\
& \left(R_{10}-1\right) \sum_{u} A_{u 2} F_{u a_{0}}+ \\
& \left(R_{20}-1\right) \sum_{u} A_{u 3} F_{u a_{0}}
\end{aligned}
$$

and

$$
\begin{aligned}
T_{a i}= & R_{0 i} \sum_{u} A_{u 1} F_{u a_{0}}+ \\
& R_{1 i} \sum_{u} A_{u 2} F_{u a_{0}}+ \\
& R_{2 i} \sum_{u} A_{u 3} F_{u a_{0}} ; \quad i=1,2,3
\end{aligned}
$$


In a similar manner the following equations for $v=$ $b, c, d, e$, and $f$ are also obtained:

$$
q_{v}=\sum_{i=1}^{3} T_{v i} \Phi_{i}
$$

The coefficients $T_{v i}$ in the above equations are called the generalized harmonic average transmissibility factors. Full expressions for the $T$ terms are given by Verma (1996a).

Equations like Eq. 16 are obtained for each tetrahedron associated with node $i$. As is evident from the derivation, the transmissibility matrix does not depend on the potentials and is a function of the $x, y$ and $z$ locations of the points $0-3$, $a, b, c, d, e, f$ and $g$ and the permeability tensors in regions 0 3 . All the flow terms for node $i$ for each tetrahedron are then collected and the node conservation equation is formed:

$$
\sum_{j=1}^{n_{c}}\left[\sum_{k=1}^{n_{s c}}\left(\sum_{l=1}^{n_{s c, n}} T_{k l} \Phi_{l}\right)\right]_{i}=\text { Accumulation }
$$

where $n_{c}$ refers to the number of connections for a grid node, $n_{s c}$ refers to the number of subconnections of a connection and $n_{s c, n}$ refers to the number of nodes which affect flow for a subconnection. A connection in this work is a link between two nodes. In the proposed method, flow along a connection is determined by the potentials at all nodes which are at the vertices of tetrahedra, of which the connection is an edge. The LHS of Eq. 17 corresponds to flow along all the connections associated with a grid node. Figure 8 shows a very simple two-dimensional example of nodes and the connections associated with the nodes. All the connections associated with node $i$ are shown as continuous lines.

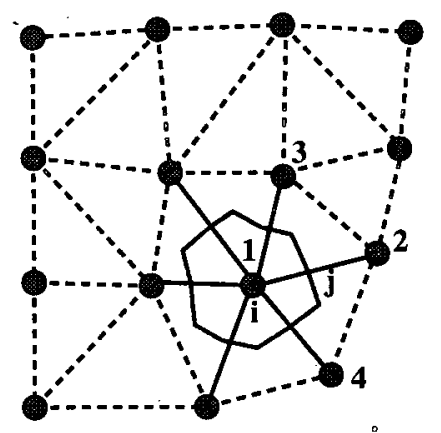

Figure 8: An example of connections network

Other Two- and Three-dimensional Geometries. Derivation for the transmissibility matrix for two-dimensional Voronoi and CVFE grids are done in a similar manner. Figure 9 shows the constraint points and boundaries in a triangle. The potential and velocity constraint points $a, b$, $c$ shown in the figure are located at the center of gridblock face. These points are used to derive transmissibilities for Voronoi and CVFE grids. A detailed derivation is given by Verma (1996a). A similar approach can be used for quadrilateral grids (Figure 9) as shown by Avatsmark et al. (1995). Edwards and Rogers (1994), presented a similar approach for quadrilateral grids. Instead of constraining the fluxes in a direction normal to any surface, they constrained the $x$ and $y$ direction fluxes across the interfaces. There can be six independent constraints for a quadrilateral (from eight possible). out of which they used only four (because they had to solve for four face pressures). Thus they used constraints which were not unique and so obtained a nonunique solution for heterogeneous systems. The derivation of transmissibilities in a quadrilateral is given by Verma (1996a). In an identical manner, transmissibilities can be obtained for hexahedra in three-dimensions. For all these geometries, the conservation equation can be put in the form of Eq. 17 which makes the computer implementation of all these grids identical.

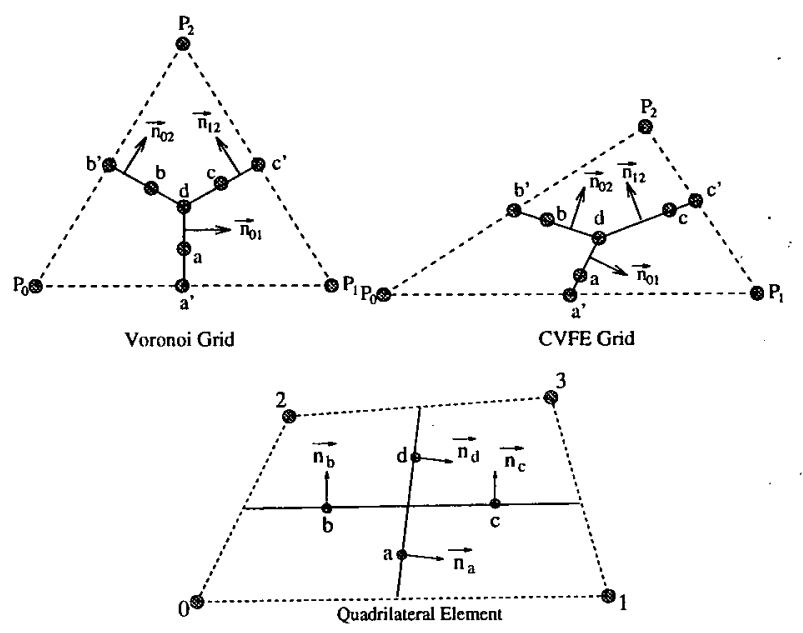

Figure 9: Location of potential and velocity continuity points for (a)Top left: Voronoi, (b) Top right: CVFE and (c)Bottom: quadrilateral grid

Extension to Multiphase Flow. For multiphase flow the phase velocity vector is written as:

$$
\vec{v}_{p}=-\lambda_{p} \overline{\bar{k}} \vec{\nabla} \Phi_{p}
$$

Assuming that the same phase mobility can be used on both sides of the interface to obtain the phase velocity constraint equations (as is normally done with upstream weighting of phase mobility), Eq. 17 can be extended to obtain conservation equations for each component in the porous medium:

$$
\begin{aligned}
& \sum_{j=1}^{n_{c}}\left[\sum_{k=1}^{n_{s c}}\left\{\lambda_{p_{i j, k}}\left(\sum_{l=1}^{n_{n}} T_{k l} \Phi_{p, l}\right)\right\}\right]_{i}= \\
& \frac{V_{i}}{\Delta t}\left[\left(\frac{\phi S_{p}}{B_{p}}\right)^{n+1}-\left(\frac{\phi S_{p}}{B_{p}}\right)^{n}\right]_{i} .
\end{aligned}
$$

In the above equation the transmissibility terms are the same as those in Eq. 17. The discretized form of the accumulation term has been used in Eq. 19. The superscripts $n$ and $n+1$ on the right-hand-side (RHS) of Eq. 19 refer to time levels of the variables. All the variables on the LHS of Eq. 19 are at time level $n+1$, hence the superscript has not been used on the LHS.

As is evident from Eq. 17, in this formulation (as in the case of CVFE), flow at each connection does not depend just on fluid potentials at the connection's nodes. Because of this, it takes more time (as compared to a generalized PEBI grid) to assemble the Jacobian matrix for the proposed method. But the structure of the Jacobian matrix is identical to that for the generalized PEBI scheme. Since most of the time (normally $80-90 \%$ ) in any simulation is taken up by the solver, the computer time of the current 
scheme will not increase significantly over GPEBI. Upstream evaluation of the mobility at any surface is based on the sign of

$$
\sum_{l=1}^{n_{s c, n}}\left(T_{k l} \Phi_{p, l}\right) \text {. }
$$

which determines the flow direction. This function is a linear combination of the potentials affecting flow across a surface. In three-dimensions, a subconnection flow is a function of four potentials.
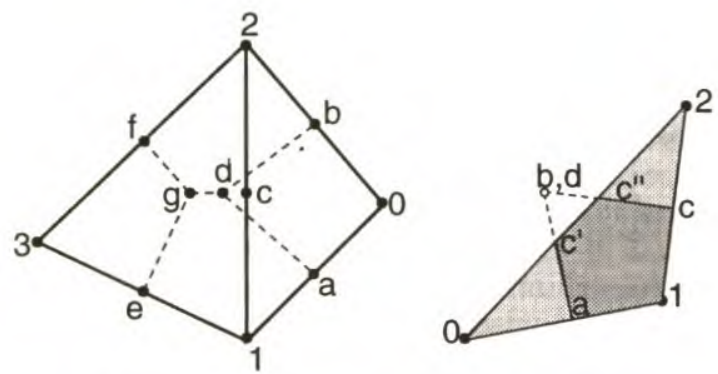

Figure 10: Approximation used when circumcenter falls outside triangle (a)Left: Case 1 (b)Right: Case 2

Special Cases for two-dimensions: Circumcenter Outside Triangle. The two-dimensional derivation follows identical steps as shown in the last section. During the derivation it is assumed that each connection makes contributions to flow in each of the tetrahedra of which the connection is a part. For Voronoi grids in two-dimensions, this is true if the circumcenter of the triangle is inside the triangle. For the case when the circumcenter falls outside a triangle, but is inside an immediate neighboring triangle, a simple approximation is possible. Figure 10 shows such a case. In this case the circumcenter of $\triangle_{012}$, point d, lies inside $\triangle_{123}$. Properties inside the triangular region 1-a-d are the same (even though this region belongs to two Delaunay triangles, viz., $\triangle_{012}$ and $\triangle_{123}$ ) because of the assumption that gridblocks have uniform properties. Hence, one can use points $a, b, d$ as potential constraint points, in the manner mentioned earlier, to estimate the transmissibilities for $\triangle_{012}$. But the transmissibility between nodes 1 and 2 in $\triangle_{012}$ will be zero because there is no gridblock face on edge 1-2 in this triangle. For $\triangle_{123}$ the transmissibility between nodes 1 and 2 is based on the length of segment $g-d$.

Figure 10 also shows the case of a boundary triangle whose circumcenter falls outside the domain. Potential is constrained at points $a, b$, and $c$. There is no flow between nodes 0 and 2 . The area available for flow between nodes 0 and 1 is determined by segment $a-c^{\prime}$ while that for flow between nodes 1 and 2 is determined by segment $c^{\prime \prime}-c$. This method of computing transmissibility will not work if the circumcenter of a triangle does not even lie inside an immediate neighboring triangle. For two-dimensions, it is very easy to locate nodes so that this condition is never violated. In three-dimensions, satisfying this condition may be difficult. However, a point to keep in mind is that nowhere during the derivation any assumption was made which would constrain the location of potential constraint points. Hence the grids used need not be of Voronoi type. Thus gridding schemes such as CVFE, or CVFE-Voronoi-BAG can be easily used.

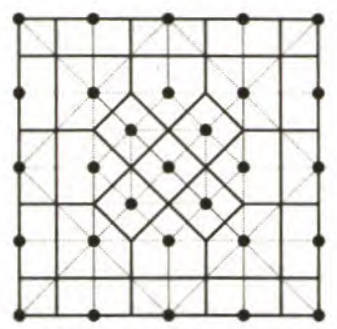

Figure 11: Description of a grid

\section{GRID CONSTRUCTION}

Introduction. There are a number of situations where Cartesian grids are unable to handle the geometry of the reservoir as well as the spatial variation of physical properties of rock and fluids in the reservoir. Flexible grids can be used in such situations. This section discusses a procedure to construct such grids. The major steps are (a) location of gridnodes, (b) Delaunay triangulation and connectivity of gridnodes, and (c) construction of gridblock geometry. Different types of gridblock geometries can be generated depending on the desired grids flexibility.

Grid Description. Solution of the primary unknowns (typically pressure and phase saturation/composition) are sought at specific points in the domain of interest. Such points will be called gridnodes in this work. Gridblocks are constructed around such gridnodes. A grid is defined as a group of such gridblocks. A gridblock is made up of a number of faces and a face is represented by an ordered set of points. In this work, properties such as porosity and permeability are assumed to be constant inside a gridblock. Other variables such as pressure and saturation are specified at the gridnodes.

The geometrical aspects of a two-dimensional grid used in this work are illustrated in Figure 11. The gridnodes are shown by the thick dots. The continuous lines denote the boundaries of a gridblock while the dotted lines denote the mesh of connections. A connection is defined as a link between two nodes. Fluid flow between two gridnodes is modeled as flow along the connection between them.

The mesh of connections in Figure 11 is obtained by a Delaunay triangulation of the gridnodes. A twodimensional triangulation means dividing the reservoir into triangles with the gridnodes at the vertices of the triangles such that the triangles fill the reservoir. Such a triangulation is said to be Delaunay when the circumcenter (i.e. the circle passing through the vertices of the triangle) of any triangle does not contain any other gridnode inside it. In three-dimensions the reservoir region is decomposed into tetrahedra such that the reservoir volume is completely filled. Such a triangulation is said to be Delaunay when the circumsphere (i.e. the sphere passing through the vertices of the tetrahedron) of each tetrahedron does not contain any other gridnode.

Location of Gridnodes. Gridnodes are to be located in the reservoir domain where the solution of the unknowns (pressure and saturation/composition) is sought.

Use of Two-Dimensional Modules. Palagi and Aziz (1991) proposed a method of locating the gridnodes for the generation of two-dimensional Voronoi grids. They defined a number of modules which had predefined loca- 
tion of points in a local coordinate system. Some of the modules used were (a) Cartesian, (b) radial, and (c) hexagonal. Location of gridnodes in each of these modules and the resulting Voronoi grid geometry are shown in Figure 12. This figure also shows a parallel Cartesian module and a tilted hexagonal module. These modules can be scaled, rotated and then placed in any particular location in the reservoir using simple coordinate transformations. This is done to easily achieve the desired gridblock geometry in different regions of the reservoir.

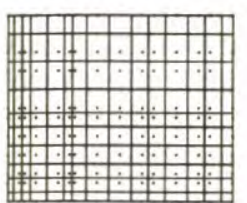

Cantesian

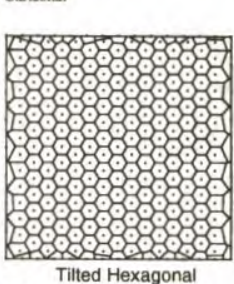

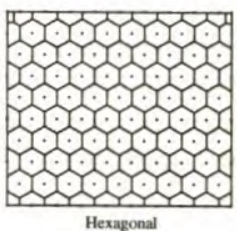
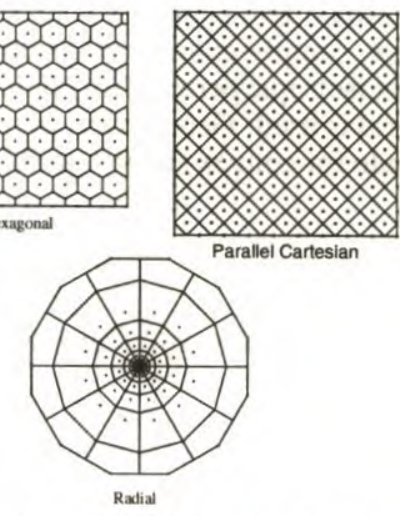

Figure 12: Modules used for grid generation in twodimensions

One can start with a base grid, which may be hexagonal or Cartesian, for example, and which covers the entire physical domain. Other modules may then be put on top of the base grid at any desired location. The gridnodes which already exist inside the area where the new module is placed are removed. Figure 6 shows a Voronoi grid whose gridnodes were generated using this procedure. The base grid is hexagonal. Radial and Cartesian modules are put in different regions.

Use of Three-Dimensional Modules. The modules mentioned in the previous section can be used to generate, what we refer to as, $2 \frac{1}{2} \mathrm{D}$ grids where stacks of two-dimensional grids are used to model vertical variation in reservoir properties. For three-dimensional grid two more modules are defined in this work. They are Cartesian and cylindrical.

Figure 13 shows a three-dimensional Cartesian module and a three-dimensional cylindrical module. Figure 14 shows a three-dimensional Voronoi grid constructed using one Cartesian module and two cylindrical modules.

The modules described in this section are only used to generate the gridnode locations. A mixed module, which is a set of arbitrarily located gridnodes, is also used in this work. Gridblocks around these nodes have to be constructed for the assignment of rock and fluid properties. Palagi and Aziz (1991) present a procedure to construct Voronoi gridblocks directly from the node locations obtained using modules. A Voronoi grid is actually the dual of Delaunay triangulation. In this work, instead of directly generating Voronoi grid geometry, Delaunay triangulation of nodes is done first. This gives the flexibility to also use several other types of grids without any significant effort.

Delaunay Triangulation. Existing numerical schemes for flexible grid and the ones presented in this paper approximate potential gradients at gridblock boundaries based on values at the vertices of a triangle (tetrahedron in threedimensions). It is thus important that the triangulation of
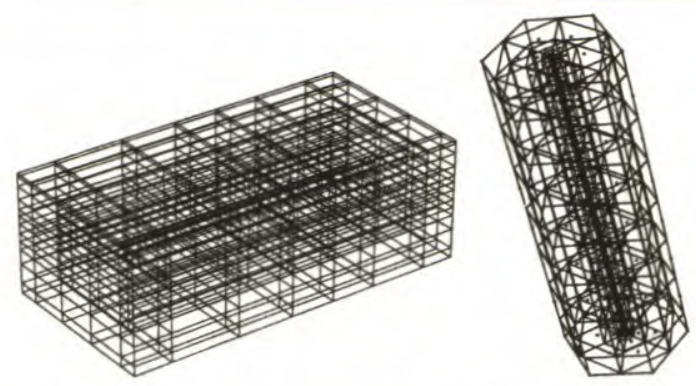

Figure 13: Three-dimensional Cartesian and cylindrical modules

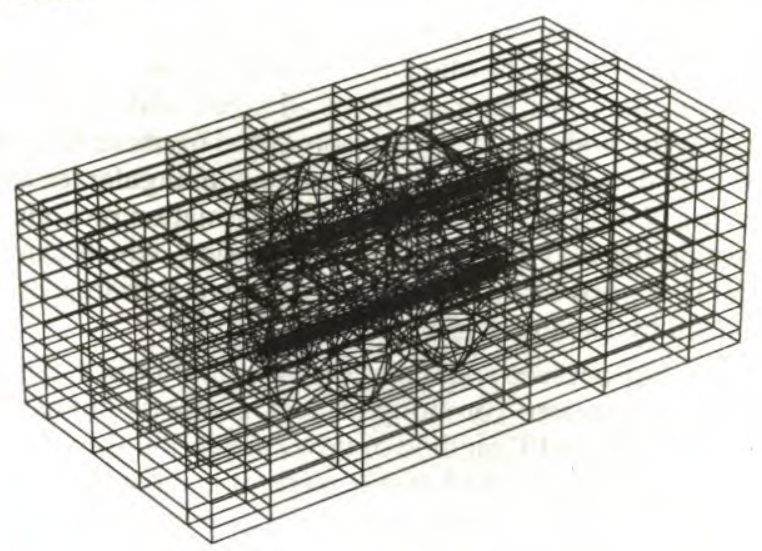

Figure 14: A three-dimensional Voronoi grid constructed using one Cartesian module and two cylindrical modules

node locations be optimal in selecting the nearest natural neighbor.

In two-dimensions Delaunay triangulation assigns triangles by the criterion that no vertex of a triangle lies inside the circumcircle of any other triangle (circle criterion). In three-dimensions it is the circumsphere of any tetrahedron that must not contain the vertex of any other tetrahedron (sphere criterion). This produces a unique triangulation, except in the case of degeneracies. Delaunay triangles define nearest natural neighbors in the sense that nodes are closer to their mutual circumcenter than are any other nodes. These circumcenters form the vertices of the Voronoi tessellation. Hence, Voronoi polygon surrounding a node is a region that is closer to that node than to any other node in the set.

In two-dimensions, Delaunay triangulation maximizes the minimum of the six angles in any pair of two triangles which make up a convex quadrilateral. This is called the max-min angle criterion (Joe, 1989). It can be shown that a Delaunay triangulation in two-dimensions which satisfies the circle criterion also satisfies the max-min angle criterion. As a result, triangles are as equiangular as possible. Even then it is quite common to get triangles which have their circumcenters outside even their neighboring triangles. Under such conditions the numerical scheme proposed in this paper cannot be used with Voronoi grids. However it is possible to add additional points which would result in acute angle triangles which satisfy the circle criterion (Baker, 1987).

A tetrahedron contains twelve planar angles (three in each of the four triangular faces), six dihedral angles (one at each of the six edges) and four solid or trihedral angles at the vertices. Gasson (1983) gives equations to 
determine these angles. Joe (1989) introduced the max-min solid angle criterion for three-dimensional triangulation. A triangulation satisfies the max-min solid angle criterion if, over all possible triangulations of the given points, the minimum of the solid angles at all vertices of all tetrahedra is maximized. In three-dimensions, Delaunay triangulation of a set of points is a triangulation that satisfies the sphere criterion. However, the three-dimensional Delaunay triangulation does not in general satisfy the max-min solidangle criterion (Joe, 1991) and does not seem to satisfy any optimal angle condition. Thus there may be tetrahedron of poor shape in three-dimensional Delaunay triangulation and 'sliver' elements may result (Joe, 1991). Slivers are tetrahedra with small solid and dihedral angles. Such sliver elements may not connect truly adjacent node locations because the node locations are connected by more than the optimum distance obtained from more equiangular tetrahedra. Joe (1991) proposes a local transformation procedure, based on the max-min solid angle criterion, which can be used as a postprocessing step to improve a triangulation obtained by Delaunay triangulation.

A postprocessed triangulation as just described cannot be used to generate Voronoi gridblock geometry but can be used with the CVFE method of flow calculation. Moreover, for the problems studied in this work, it is most likely that a three-dimensional triangulation will give some tetrahedra whose circumcenter falls outside even its immediate neighbors. When a three-dimensional Voronoi grid is formed from such a triangulation, the proposed numerical scheme does not work. In three-dimensions, a triangulation, which always satisfies such a criterion and is still able to produce Voronoi gridblock geometries which conform to required physical boundaries and other desired geometrical shapes, is almost impossible to generate. However a grid, in which Voronoi, CVFE and BAG grids are mixed to satisfy the above-mentioned requirements, can be used without any problem to model flow.

Connectivity. All of the gridding procedures studied here use Delaunay triangulation as the first step. The geometrical objects which appear are nodes, edges, triangles and tetrahedra. Each object occurring in the system is assigned a unique reference number. An edge is defined by the two nodes it connects, a triangle by the three nodes it connects, and a tetrahedron by the four nodes it connects. Furthermore, for calculations to compute gridblock geometry, it is essential to establish how these objects connect to each other. The generation of such connectivity information may become quite expensive if it has to be done a number of times during the simulation process. In this work, since grid geometry is generated only once for a simulation, no attempt was made to make the grid generation algorithms numerically very efficient.

Construction of Flexible Grids Once the Delaunay triangulation of the nodes is available, flexible grids can be easily constructed. Voronoi, GPEBI and CVFE grids fall in this category.

Voronoi Grids. Given a set of gridnodes, gridblocks can be generated which satisfy the Voronoi criterion. As already mentioned a Voronoi control volume (gridblock) is defined as the volume associated with each gridnode which is nearer to its own gridnode than to any other gridnode. Figure 15 shows a Voronoi control volume associated with gridnode 0 . Intersections of perpendicular bisector lines (planes in three-dimensions) of the Delaunay mesh of connections define the boundaries of the gridblock: the perpendicular bisectors of each triangle intersect at its circumcenter, for example, in Figure 15, lines $c_{0}-e_{0}, c_{0}-e_{1}$ and $c_{0}-e_{2}$ intersect at point $c_{0}$. The faces of a Voronoi gridblock lie on these perpendicular bisectors of the connections; the circumcenters form the vertices of these faces, e.g. points $c_{0}, \ldots, c_{5}$ are vertices of Voronoi block of node 0 . Because of the way the Voronoi grid geometry is generated, it always gives convex (i.e. simply connected) gridblocks. The numerical model for isotropic permeability field is very simple when Voronoi grid is used. Geometrical properties are also easier to compute for convex shaped gridblocks (Verma, 1996b).

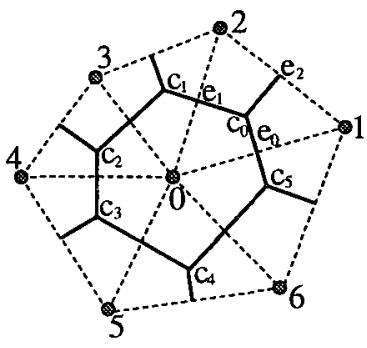

Figure 15: Voronoi gridblock for node 0

A Voronoi grid is defined to be simple (in this work) when each Delaunay triangle's circumcenter lies inside the triangle or inside one of its immediate neighboring triangles. For such cases, Voronoi grid can be simply constructed using the edge-centers and the circumcenters. The number of faces the gridblock will have depends on the number of edges connected to the node, the number of boundary edges connected to the node and the number of boundary edges in the triangles connected to the node.

A Voronoi gridblock description is constructed by collecting the information of face descriptions from each Delaunay triangle. Consider Figure 16a. Assume that triangle $0-1-2\left(\triangle_{012}\right)$ is in the interior of a physical domain. Node 0 has two faces in this triangle. One of them is $c_{0}-e_{0}$ and the other is $c_{0}-e_{1}$. Similarly, each of the nodes 1 and 2 have two faces in this triangle. Now, if the circumcenter falls outside a boundary edge of a triangle then the gridblock faces are constructed as shown in Figure $16 \mathrm{~b}$. Here the edge 0-2, falls on the boundary of the physical domain. The gridblock of node 1 is made of faces $e_{0}-x_{1}, x_{1}-x_{2}$ and $e_{2}-x_{2}$, where $x_{1}$ and $x_{2}$ are intersections of the boundary edge $0-2$ with perpendicular bisectors $e_{0}-c$ and $e_{2}-c$, respectively. Faces of gridblocks of nodes 0 and 2 are also shown in the figure.

Figure 16c shows a case where the circumcenter of $\triangle_{123}$ falls outside this triangle, but is still inside the physical domain and also inside a neighboring triangle (i.e $\triangle_{012}$ ). In such a case node 1 has the faces described by three line segments, viz. $e_{0}-c_{0}, c_{0}-c_{1}$ and $e_{3}-c_{1}$. Point $e_{2}$ does not fall on any face. Faces of gridblock associated with the other nodes are also shown in the figure.

Figure 16d shows 4 nodes and their triangulations. Each triangle has an edge at the boundary and each of them has its circumcenter outside the physical domain. Hence, none of the circumcenters and edge-centers form any part of any gridblock face. The face vertices are given by the intersection of the perpendicular bisectors with the boundary edges. All the faces are shown by continuous lines. 

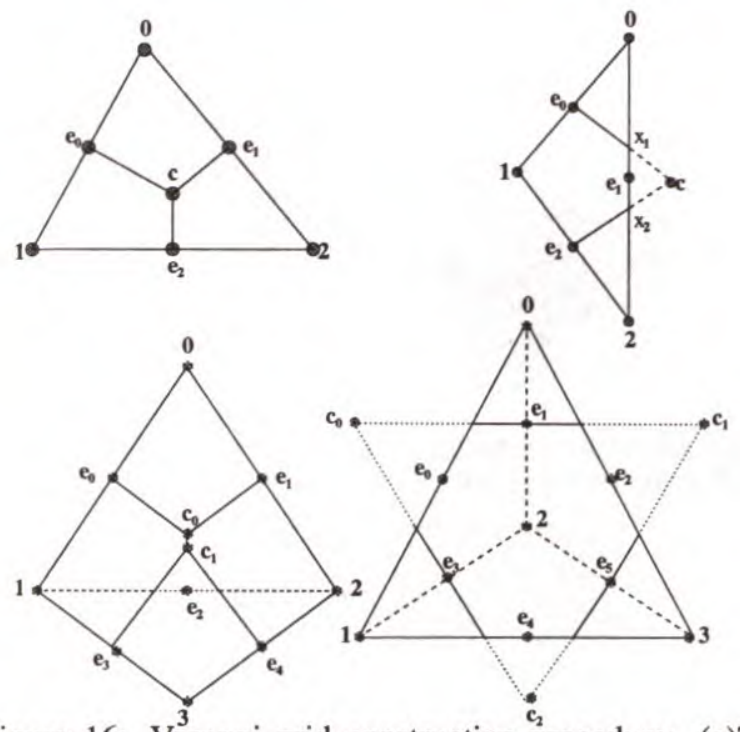

Figure 16: Voronoi grid construction procedure: (a)Top Left: circumcenter inside triangle, (b)Top right: circumcenter outside boundary (c)Bottom left: circumcenter inside another triangle (d)Bottom right: all circumcenters outside boundary

Examples of Voronoi Grid. Figure 17 shows an example of two-dimensional Voronoi grids where gridblock boundaries have been aligned along reservoir bed boundaries, inclined well and a fault. Nodes in this case were located using a mixed module. They are equidistant from the bed boundaries on lines normal to bed boundaries. Cartesian modules were then placed along the well axis.

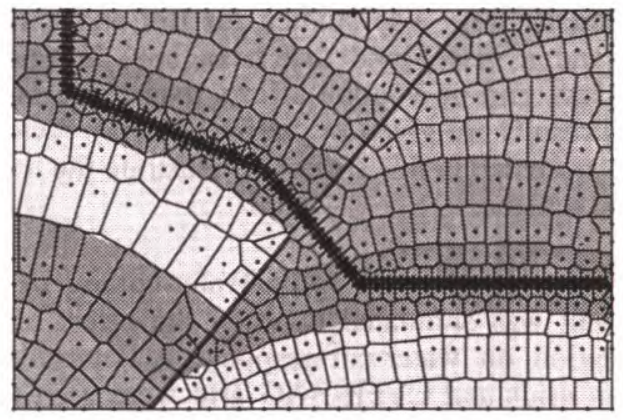

Figure 17: Voronoi grid along deviated well, layer boundaries and fault

Figure 18 shows radial grids near a well. A Cartesian module was used as the base grid. A radial grid with the origin exactly at the well location was then placed after removing all existing points which fell inside the radial module.

Figure 19 shows a $2-\frac{1}{2} \mathrm{D}$ Voronoi grid. Grids of the same geometry in $x-y$ plane were stacked to model vertical variations in reservoir properties. The bottom figure shows horizontal gridblock faces along with some of the gridblocks around vertical wells. A three-dimensional Voronoi grid with cylindrical grids around two wellbores was shown in Figure 13.

CVFE Grids. A two-dimensional grid is defined to be of CVFE type if gridblock boundaries are made from the edgecenters and barycenter of Delaunay triangles. Barycenter of a triangle is the intersection point of lines from a vertex

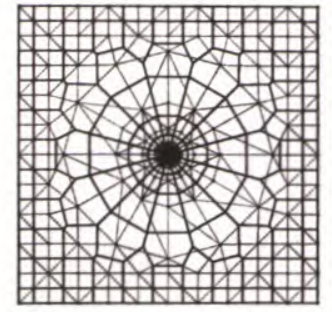

Figure 18: Two-dimensional Voronoi gridblocks around a well

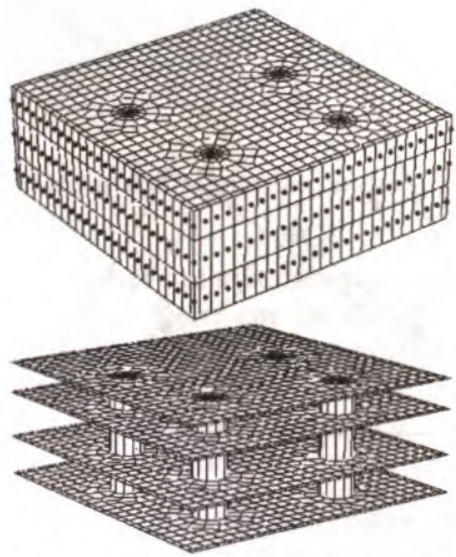

Figure 19: A 2- $\frac{1}{2} \mathrm{D}$ Voronoi grid

to the center of opposite edge. Figure 20a shows the edgecenters (points $\mathrm{e}_{0}, \mathrm{e}_{1}$ and $\mathrm{e}_{2}$ ) and the barycenter (point $\mathrm{c}$ ) for $\Delta_{012}$. There are three gridblock faces inside each triangle, i.e. one for each edge. These faces describe the CVFE gridblock associated with each node, e.g. the face between nodes 1 and 2 on edge $1-2$ is $c-e_{2}$.

In three-dimensions, a CVFE grid is made from the edge-centers, barycenters of the triangular faces of the tetrahedra and barycenter of the tetrahedra. For a tetrahedron, the lines from its nodes to the barycenters of the opposite triangle faces intersect at a common point. This point is the barycenter of the tetrahedron. In Figure 20b. $e_{0}$, $\ldots, e_{5}$ are edge centers, $c_{1}$ is barycenter of $\Delta_{013}$ and $d$, the intersection point of lines $1-c_{2}$ and $2-c_{1}$, is the barycenter of the tetrahedron. There are six gridblock faces inside each tetrahedron, one on each edge. These faces describe the CVFE gridblock associated with each node, e.g. the face between nodes 1 and 3 on edge 1-3 is made from the points $c_{1}, e_{4}, c_{3}$, d. This face forms a part of a face of gridblocks associated with nodes 1 and 3 .

CVFE gridblocks are normally not convex (unlike Voronoi gridblocks which are always convex), which complicates the calculation of some of the geometrical properties. It is assumed in this work that an upscaling procedure is available (e.g. Tran and Journel, 1995) which can be used to obtain coarse scale permeability distribution for nonconvex gridblocks.

The procedure to construct a CVFE grid is quite simple. Complications occur because gridblock face vertices have to be arranged in the correct order. This enables geometrical characteristics of the gridblock, such as its volume and face areas, to be easily and correctly computed. It also allows visualization programs to correctly interpret and plot the gridblock data.

Examples of CVFE Grid. A two-dimensional CVFE grid is shown in Figure 21. It is very difficult to visualize grid- 

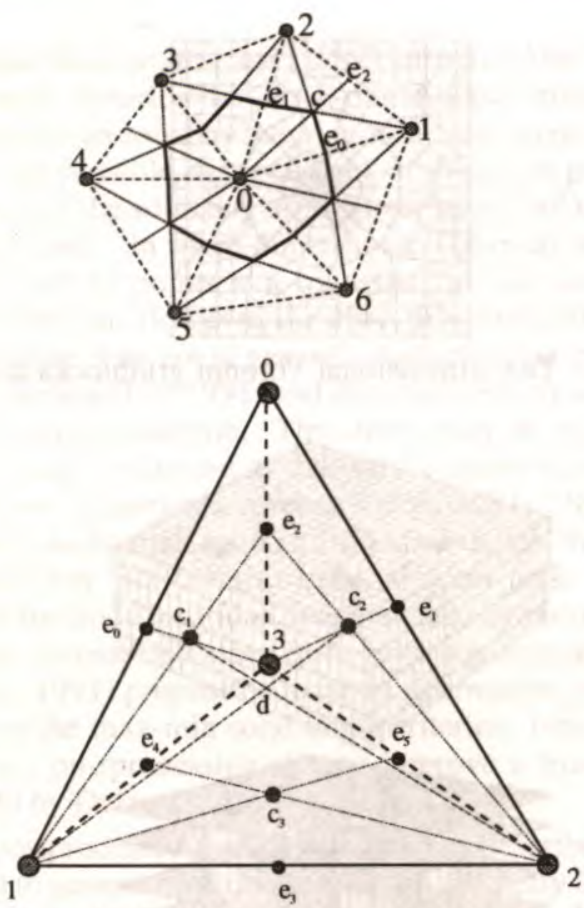

Figure 20: Edge-centers and barycenters for (a)Top: Threedimensional CVFE grid construction (b)Bottom: Threedimensional CVFE grid construction

block geometry of CVFE grids in three-dimensions. Hence, only the Delaunay triangulation of the nodes is shown in Figure 22. This figure shows an inclined well in the interior of the domain. Gridnodes were generated using a Cartesian base module and a cylindrical module.

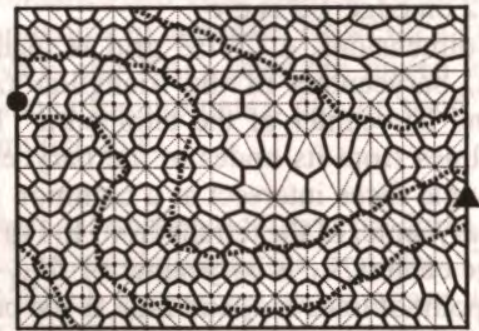

Figure 21: A two-dimensional CVFE grid example

CVFE-BAG Grid. Gridblocks in the case of CVFE grids were completely described by points on edges (i.e. edgecenters) and a point inside the triangle (i.e. barycenter of the triangle). These points can be slightly shifted so that the gridblock boundaries get aligned with reservoir heterogeneities. This is achieved in two steps:

\section{Form a CVFE grid.}

2. For all the triangles which are intersected by a bed boundary, the intersection points, instead of the edgecenters and barycenter/circumcenter are used to form gridblock faces inside that triangle.

Figure 23 shows an example of a two-dimensional CVFE-BAG grid.

Generating such a grid in three-dimensions is geometrically very difficult and requires complex algorithms and computations. A very simple case of CVFE-BAG grid whose grid block boundaries are modified to align along a planar physical boundary such as a water-oil contact or a fault has been implemented in this work.

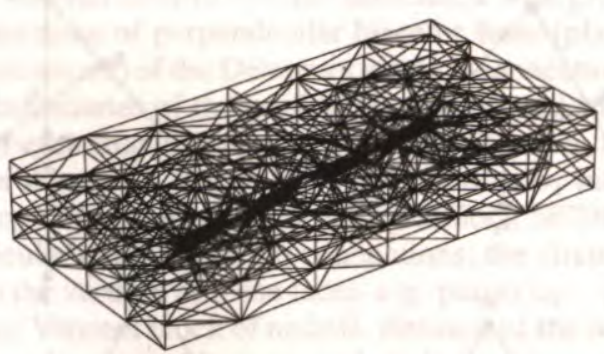

Figure 22: Three-dimensional triangulation of nodes for CVFE grid construction

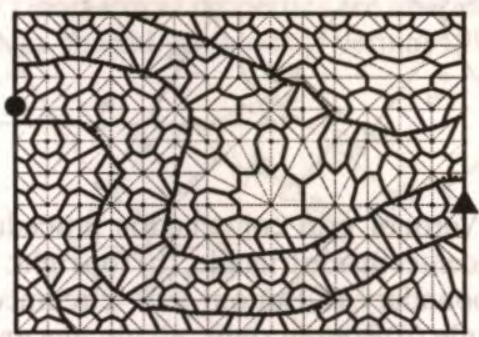

Figure 23: A two-dimensional CVFE-BAG grid example

\section{NUMERICAL TESTS AND APPLICATIONS}

Some single-phase and two-phase flow simulation test runs with the proposed numerical scheme are described in this section. A number of tests were carried out to study the effect of anisotropy and grid-orientation on the pressure response around a well. It was observed that for full and anisotropic tensors, hexagonal Voronoi grids were more accurate than Cartesian grids. Adverse mobility ratio displacements were also studied for full and anisotropic tensors and it was found that hexagonal Voronoi grids showed less grid-orientation effects than Cartesian grids. Detailed results are given by Verma (1996a). Two examples are presented in this section to illustrate the use of the proposed scheme.

Coning in a Horizontal well. Water-coning in a horizontal well was simulated to study the effect of spatial discretization on water-cut response. Point-distributed Cartesian grids and CVFE-BAG grid were used to study this problem. For static grids, a close spacing of gridnodes near wellbore regions results in fewer gridblocks than in conventional grid to achieve desired accuracy.

The problem studied in this section is very similar to the one studied by Consonni et al. (1992). Only a homogeneous reservoir permeability is considered. The value of permeability is $360 \mathrm{md}$ in the horizontal direction and $60 \mathrm{md}$ in the vertical direction. The reservoir has a thin oil column of $35 \mathrm{~m}$ thickness. The water zone is $195 \mathrm{~m}$ thick. The horizontal well is located $25 \mathrm{~m}$ above the water-oil contact. A water injector is use to maintain the pressure in the aquifer. The section studied is $600 \mathrm{~m}$ long and $300 \mathrm{~m}$ wide. Some of the essential reservoir and well geometry data and well production data are given in Table 1 .

The Eclipse simulator from Geo-Quest was used to generate reference finite-difference solutions for this problem. Several Cartesian grid sizes were used. The coarsest grid used had $280(7 \times 5 \times 8)$ gridblocks and the finest used had $92659(61 \times 31 \times 49)$ gridblocks. The solution appears to be converged for the grid given by $21 \times 17 \times 49$, i.e., 25823 grid blocks. The water-cut response for some of 
Table 1: Reservoir and Well Data: Water Coning Problem

\begin{tabular}{|ll|}
\hline \hline Depth to reservoir top & $1425 \mathrm{~m}$ \\
Length of section studied & $600 \mathrm{~m}$ \\
Width of section studied & $300 \mathrm{~m}$ \\
Pay zone thickness & $35 \mathrm{~m}$ \\
Aquifer thickness & $195 \mathrm{~m}$ \\
Reservoir Pressure & $2300 \mathrm{psi}$ at $1460 \mathrm{~m}$ \\
Initial Oil in Place & $1206824.6 \mathrm{Sm} 3$ \\
Initial Water in Place & $9111297.6 \mathrm{Sm} 3$ \\
Well Locations & $\mathrm{x}=150 \mathrm{~m}$ to $450 \mathrm{~m}$ \\
Producer & $\mathrm{y}=150 \mathrm{~m}$ \\
& $\mathrm{z}=1435 \mathrm{~m}$ \\
Injector & $\mathrm{x}=300 \mathrm{~m}$ \\
& $\mathrm{y}=150 \mathrm{~m}$ \\
Injection rate & $\mathrm{z}=1565 \mathrm{~m}$ \\
Production rate & 1886.94 STB/D of water \\
\hline
\end{tabular}

these grids is shown in Figure 24. As is expected from a Cartesian finite-difference scheme, the water-front gets diffused when coarse grids are used, causing an earlier waterbreakthrough.

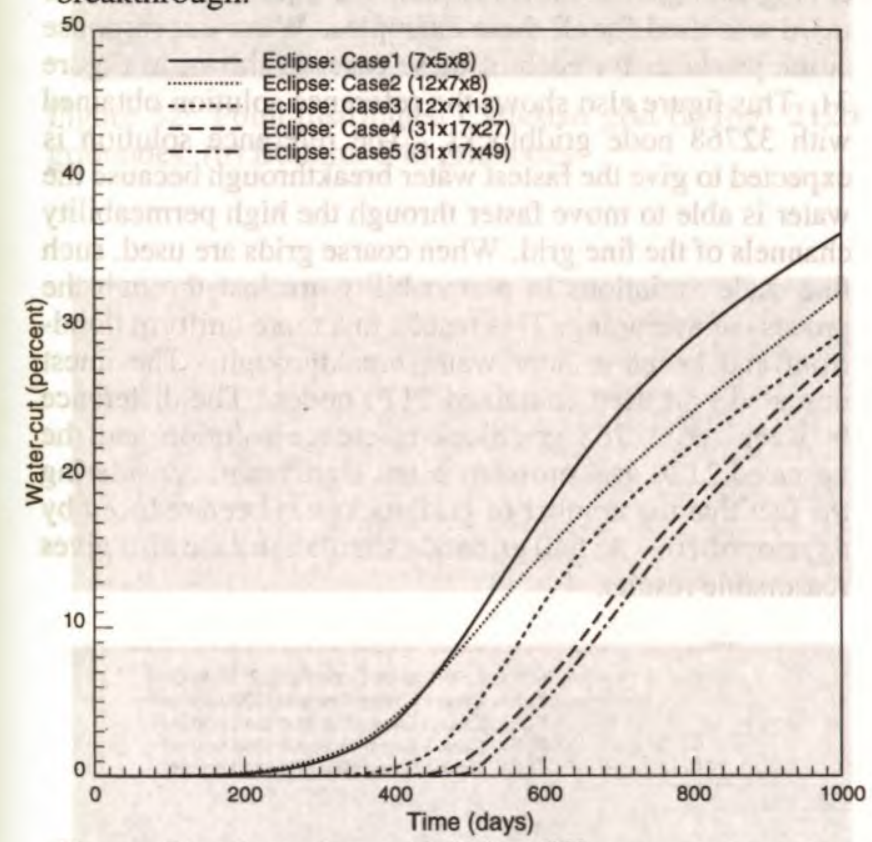

Figure 24: Cartesian grid finite difference water-cut response: Coning problem

The same problem was then studied using the 3D CVFE-BAG option of FLEX. In this case fine grids were used near both the producer and the injector. Gridnodes were located around the horizontal producer using the cylindrical module and closely-spaced gridnodes were located around the injector using the 3D Cartesian module. Only 2066 gridnodes are used in this example. Since CVFE-BAG grids are very difficult to visualize in $3 \mathrm{D}$, the 3D Delaunay triangulation of the nodes used in forming the CVFE-BAG grid are shown in Figure 25. The grid-block boundaries of the CVFE grid were modified to exactly align with the water-oil contact, hence the name CVFE-BAG grid is used here.

The water-cut response for this case is shown in Figure 26 along with the reference solution from Eclipse. It is ob-

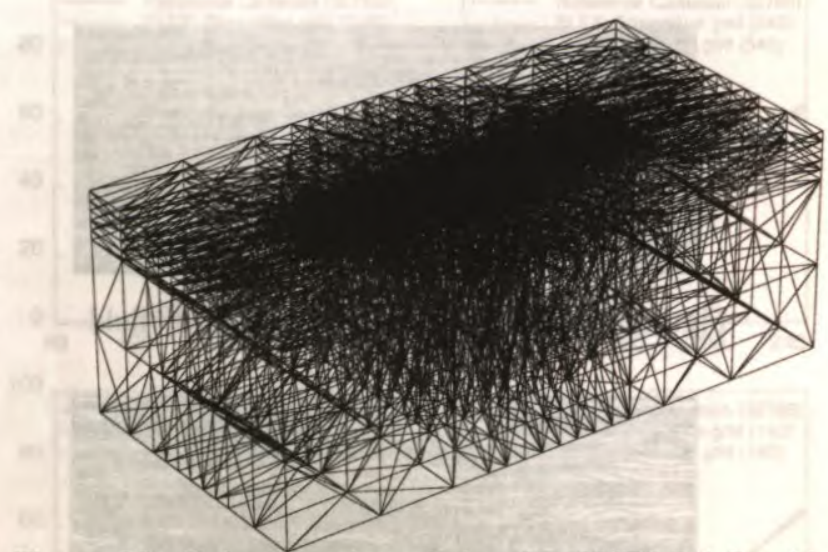

Figure 25: Delaunay triangulation for CVFE-BAG grid: Coning problem

served than the water-cut matches quite well, and is within $1 \%$ of the reference water-cut for the entire period. Consonni et al. used stacks of Voronoi grids in the vertical direction, as a result of which the same refinement had to be used in each layer. An important point to note in this example is that no such restriction exits with the 3D CVFE-BAG formulation. In fact, gridnode spacing near the producer is different from that near the injector (the injector is vertically below the center of the producer). This minimizes the number of gridnodes required for a given problem.

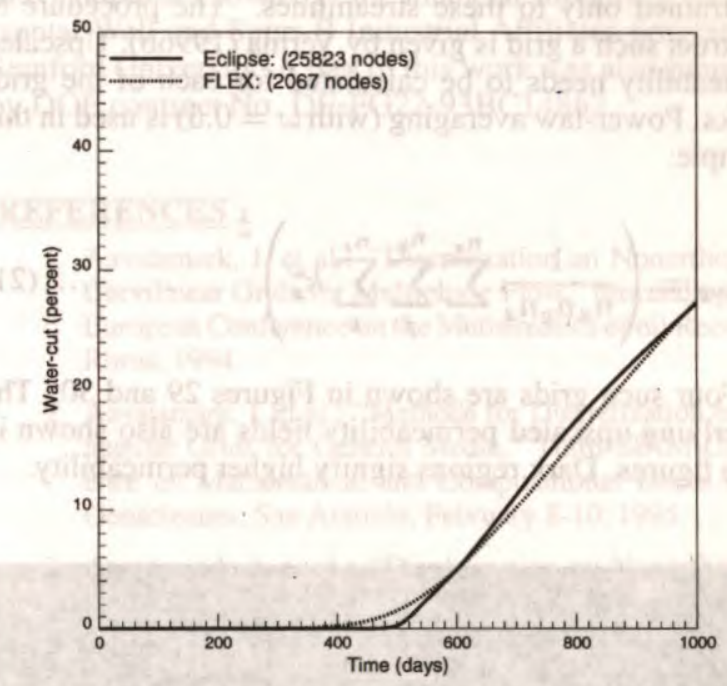

Figure 26: Comparison of FLEX (2066 node) and Eclipse reference solution for water-cut response: Coning problem

Aligning grid along stream lines. Streamlines have been used extensively to characterize flow patterns. Thiele et al. (1996) present a novel way of applying streamlines in heterogeneous systems. This example illustrates the alignment of gridblock boundaries along streamlines and its effect on the solution. Quadrilateral or triangle based flexible grids can be used for this problem. The permeability field used for this example problem is shown in Figure 27. The fine scale permeability is described on a 256 by 128 grid. i.e. 32768 nodes.

A horizontal injector is located on the left side and a horizontal producer is located on the right side. Streamlines for constant pressure boundary conditions at the two wells are shown in Figure 28. 


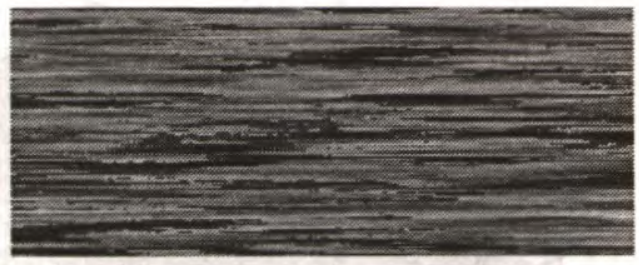

Figure 27: A heterogeneous permeability field

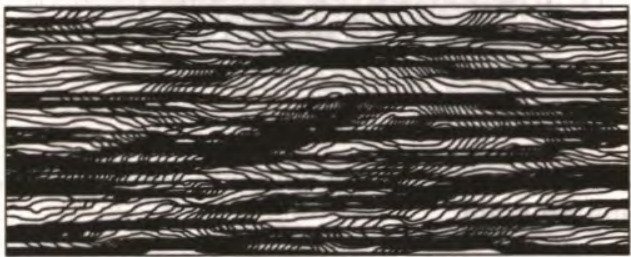

Figure 28: Streamlines for horizontal wells at both ends of Figure 27

To simulate performance of fluid flow in a reasonable time span, this permeability field has to be upscaled. The upscaling procedure can be done along the streamlines. Quadrilateral gridblocks can be constructed by aligning the gridblock boundaries along the streamlines. Since there are a large number of streamlines in the figure, streamlines are arbitrarily selected at equal intervals and gridblocks are constrained only to these streamlines. The procedure to construct such a grid is given by Verma (1996b). Upscaled permeability needs to be calculated for each of the gridblocks. Power-law averaging (with $\omega=0.5$ ) is used in this example:

$$
k_{\text {avg }}=\left(\frac{1}{n_{x} n_{y} n_{z}} \sum_{i}^{n_{x}} \sum_{j}^{n_{y}} \sum_{k}^{n_{z}} k_{x}^{\omega}\right)^{\frac{1}{\omega}}
$$

Four such grids are shown in Figures 29 and 30. The underlying upscaled permeability fields are also shown in these figures. Dark regions signify higher permeability.
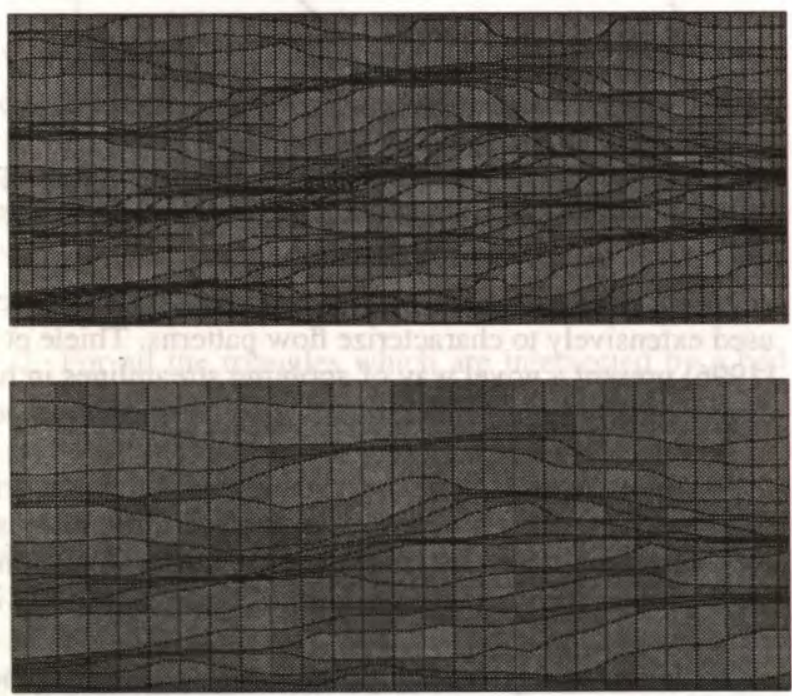

Figure 29: Example of grid aligned along streamlines (a)Top: 2120 gridnodes. (b) Bottom: 540 gridnodes
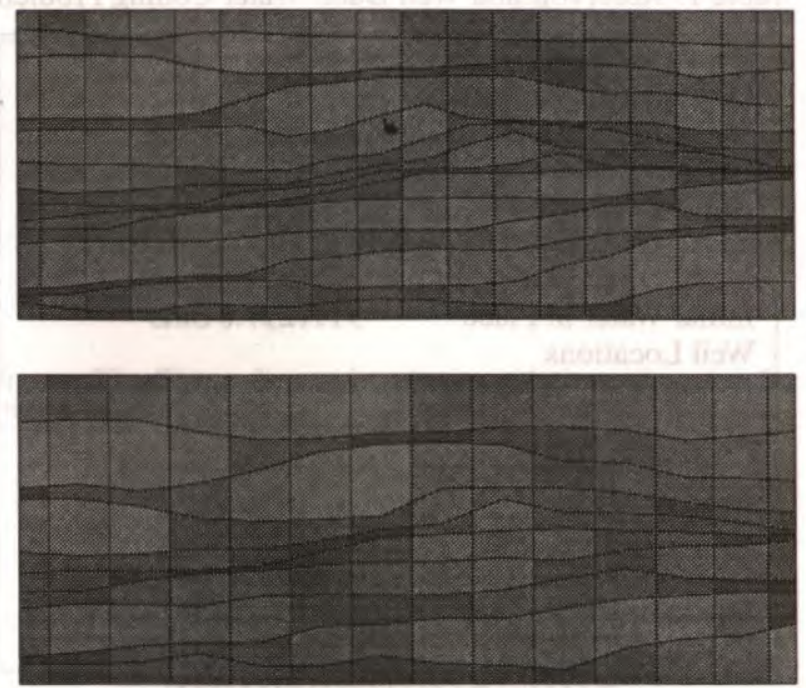

Figure 30: Example of grid aligned along streamlines (a)Top: 266 gridnodes (b) Bottom: 140 gridnodes

The rock and fluid data are the same as used in the coning example discussed earlier. An injection rate of 100 $\mathrm{m} 3 / \mathrm{d}$ was used for all these examples. Water-cut response at the producer for each of these cases is shown in Figure 31. This figure also shows the reference solution obtained with 32768 node gridblocks. The reference solution is expected to give the fastest water breakthrough because the water is able to move faster through the high permeability channels of the fine grid. When coarse grids are used, such fine scale variations in permeability are lost through the process of averaging. This results in a more uniform floodfront and hence a latter water breakthrough. The finest upscaled grid used contained 2120 nodes. The difference between the 32768 gridblock reference solution and the upscaled 2120 grid problem is not significant, considering the fact that the number of gridblocks has been reduced by a factor of 16 . The 540 gridnode simulation case also gives reasonable results.

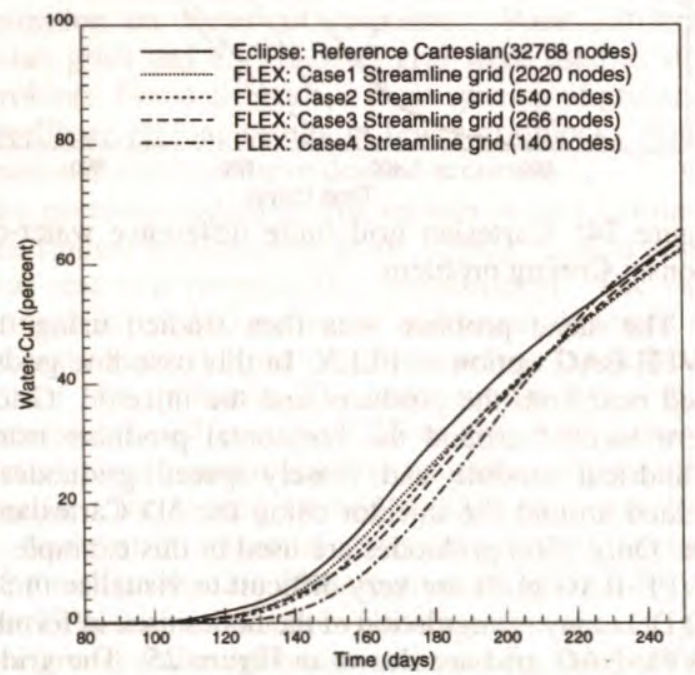

Figure 31: Water-cut of streamline grid compared with finescale response

The same four cases were then studied with conventional point-distributed grid. The upscaled permeability 
field for each of these cases is given in Figures 32 and 33. Water-cut response for each of these cases is shown in Figure 34 along with the water-cut for the streamline grids. It is evident from the figure that aligning the grid block boundaries along streamlines improves the water-cut response by a significant amount.
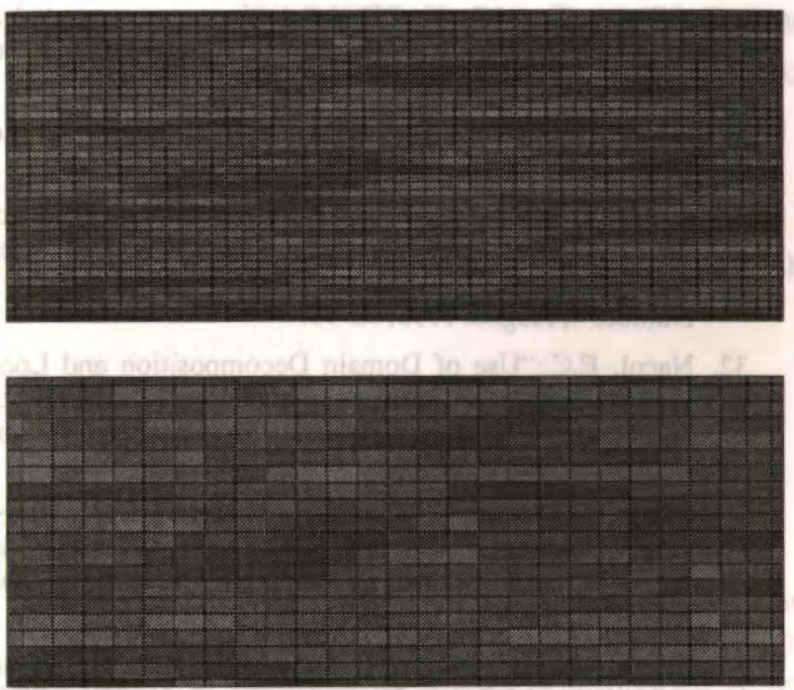

Figure 32: Point-distributed Cartesian grid (a)Top: 2120 gridnodes. (b) Bottom: 540 gridnodes
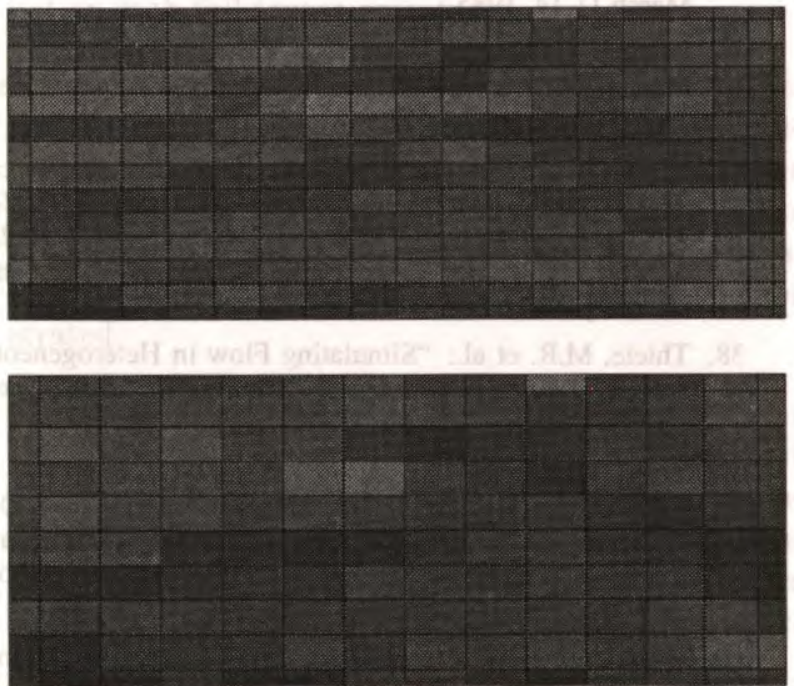

Figure 33: Point-distributed Cartesian grid (a)Top: 266 gridnodes (b) Bottom: 140 gridnodes

\section{CONCLUDING REMARKS}

Several techniques are presented in this paper for generating two- and three-dimensional flexible grids for reservoir simulations and for calculating flow over such grids. Test results show that this flexibility can be exploited to significantly reduce the number of blocks required for complex reservoir simulation problems.
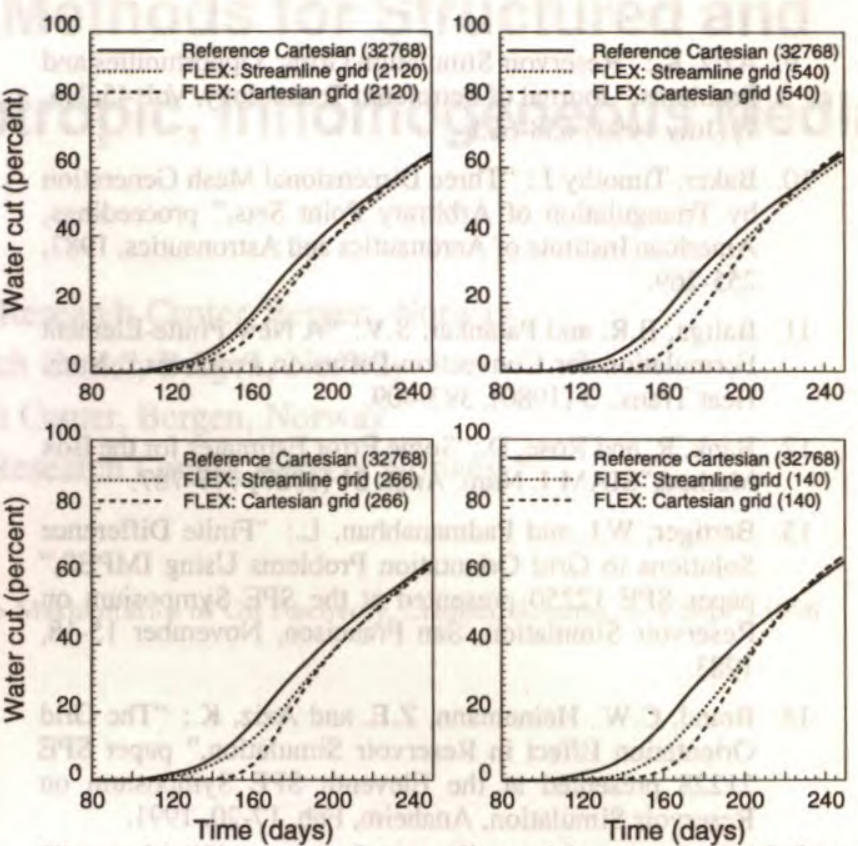

Figure 34: Water-cut of streamline grid compared with finescale and point-distributed Cartesian response (a)Top left: 2120 nodes (b)Top right: 540 nodes (c) Bottom left: 540 nodes (d)Bottom right: 140 nodes

\section{ACKNOWLEDGEMENTS}

The authors acknowledge the financial support of the Horizontal Well and Supri-B Industrial Affiliates programs of Stanford University. A part of this work was also supported by DOE contract No. DE-FG22-93BC14862.

\section{REFERENCES}

1. Aavatsmark, I. et al.: "Discretization on Nonorthogonal Curvilinear Grids for Multiphase Flow," proceedings, 4th European Conference on the Mathematics of oil Recovery, Roros, 1994.

2. Aavatsmark, I. et al.: "Methods for Discretization on Triangular Grids for General Media," Third SIAM Conference on Mathematical and Computational Issues in the Geosciences, San Antonio, February 8-10, 1995.

3. Aavatsmark, I. et al.: "Discretization on Nonorthogonal Quadrilateral Grids for Inhomogeneous, Anisotropic Media," Submitted to J. Comput. Phys (personal communication).

4. Aavatsmark, I. et al.: "Discretization on Unstructured Grids for Inhomogeneous, Anisotropic Media: Parts I and II," Submitted to SIAM J. Sci. Comput. (personal communication).

5. Amado, L.C.N., Gamzer, L., and Heinemann, Z.E.: "Finite Volume Discretization of the Fluid Flow Equations on General Perpendicular Bisection Grids," Fifth International Forum on Reservoir Simulation, Muscat, Oman, Dec 10-14, 1994.

6. Avis D. and Bhattacharya, B.K: "Algorithms for computing $d$-dimensional Voronoi diagrams and their duals," F.P. Preprate ed., Advances in Computing Research, JAI Press, Vol 1 (1983), 159-180.

7. Aziz, K. and Settari, A.: Petroleum Reservoir Simulation, Applied Science Publishers Ltd., London (1979).

8. Aziz, K.: Notes for Petroleum Reservoir Simulation, Stanford University, Stanford (1995). 
9. Aziz, K.: "Reservoir Simulation Grids: Opportunities and Problems," Journal of Petroleum Technology, Vol. 45, No. 7, (July 1993) 658-663.

10. Baker, Timothy J.: "Three Dimensional Mesh Generation by Triangulation of Arbitrary Point Sets," proceedings, American Institute of Aeronautics and Astronautics, 1987, 255-269.

11. Baliga, B.R. and Patankar, S.V.: "A New Finite-Element Formulation for Convection-Diffusion Problems," Num. Heat Trans., 3 (1980), 393-409.

12. Bank, R. and Rose, D.: "Some Error Estimates for the Box Method,"SIAM J. Num. Anal. 24 (1987) 777-787.

13. Bertiger, W.I. and Padmanabhan, L.: "Finite Difference Solutions to Grid Orientation Problems Using IMPES," paper SPE 12250 presented at the SPE Symposium on Reservoir Simulation, San Francisco, November 15-18, 1983

14. Brand, C.W., Heinemann, Z.E. and Aziz, K.: "The Grid Orientation Effect in Reservoir Simulation," paper SPE 21228 presented at the Eleventh SPE Symposium on Reservoir Simulation, Anaheim, Feb. 17-20, 1991.

15. Ciment, M. and Sweet, R.A.: "Mesh Refinement for Parabolic Equations," Journal of Computation Physics (Dec 1973) 513-525.

16. Consonni, P. et al.: "Flexible Gridding Techniques for Coning Studies in Vertical and Horizontal Wells," paper SPE 25563 presented at the SPE Middle East Oil Show, April 3-6, 1993.

17. ECLIPSE Reference Manual, Version 95a, Exploration Consultants Limited, Henley-on-Thames, UK (Jul. 1995).

18. Edwards, M.G. and Rogers, C.F: "A Flux Continuous Scheme for the Full Tensor Pressure Equation," proceedings, 4th European Conference on the Mathematics of Oil Recovery, Roros, 1994.

19. Forsyth, P.A.: "A Control Volume Finite Element Method Method for Local Mesh Refinement," paper SPE 18415 presented at 10th SPE Symposium on Reservoir Simulation, Houston, February 6-8, 1989.

20. Fung, L.S, Hiebert, A.D and Nghiem, L.: "Reservoir Simulation with a Control-Volume Finite-Element Method," paper SPE 21224 presented at 11th SPE Symposium on Reservoir Simulation, Anaheim, February 17-20, 1991.

21. Fung, L.S-K., Buchanan, W.L. and Sharma, Ravi: "Hybrid-CVFE Method for Flexible Grid Reservoir Simulation," paper SPE 25266 presented at 12th SPE Symposium on Reservoir Simulation, New Orleans, February 28-March 3, 1993.

22. Gasson, P.C.:Geometry of Spatial Forms, Ellis Horwood Limited, West Sussex, England, (1983).

23. Heinrich, B: Finite Difference Methods on Irregular Networks, Birkhauser, Basel, Boston, Stuttgart, (1987).

24. Heinemann, Z.E. and Brand, C.W.: "Gridding Techniques in Reservoir Simulation," proceedings, First and Second International Forum on Reservoir Simulation, Alpbach, Austria, September 12-16, 1988 and September 4-8, 1989, 339-426,

25. Heinemann, Z.E. and Brand, C.W.: "Modeling Reservoir Geometry With Irregular Grids," paper SPE 18412 presented at 10th SPE Symposium on Reservoir Simulation, Houston, February 6-8, 1989.

26. Heinemann, Z.E.: “Advances in Gridding Techniques," Fifth International Forum on Reservoir Simulation, Mus cat, Oman, Dec 10-14, 1994.
27. Joe, Barry: "Three Dimensional Triangulations from Local Transformations," Siam Journal of Sci. and Stat. Comput., Vol. 10, No. 4, July 1989, 718-741.

28. Joe, Barry: "Delaunay Versus Max-Min Solid Angle Triangulations for Three Dimensional Mesh Generation," International Journal for Numerical Methods in Engineering, Vol. 31, 1991, 987-997.

29. King, M.J.:"Application and Analysis of Tensor Permeability to Cross-Bedded Reservoirs", paper presented at the 7th European IOR Symposium in Moscow, Russia, October 27-29, 1993.

30. Lake, L.W.:Enhanced Oil Recovery, Prentice Hall, Englewood Cliffs, New Jersey, (1989).

31. Lim, K.T., Schiozer, D.J., and Aziz, K.: "A New Approach for Residual and Jacobian Array Construction in Reservoir Simulators," SPE Computer Applications, Volume 7, Number 4, August 1995, 93-97.

32. Nacul, E.C.:"Use of Domain Decomposition and Local Grid Refinement in Reservoir Simulation," Ph.D. Dissertation, Department of Petroleum Engineering, Stanford University, March 1991.

33. Palagi, C.L. and Aziz, K.: "Use of Voronoi Grids in Reservoir Simulation," paper SPE 22889 presented at the 66th Annual Technical Conference and Exhibition, Dallas, October 6-9,1991.

34. Palagi, C.L.: "Generation and Application of Voronoi Grids to Model flow in Heterogeneous Reservoirs," Ph.D. Dissertation, Stanford University, May 1992.

35. Pedrosa, O.A. and Aziz, K.: "Use of Hybrid Grid in Reservoir simulation," paper SPE 13507 presented at Middle East Oil Technical Conference and Exhibition, Bahrain, March 11-14, 1985.

36. Prakash, C.:"Examination of the Upwind Formulation in the Control Volume Finite Method for Fluid Flow and Heat Transfer," Num. Heat Transfer 11 (1987) 401-416.

37. Sonier, F. and Eymard, R.: "Mathematical and Numerical Properties of Control-Volume Finite-Element Scheme for Reservoir Simulation," paper SPE 25267 presented at 12th SPE Symposium on Reservoir Simulation, New Orleans, February 28-March 3, 1993.

38. Thiele, M.R. et al.: "Simulating Flow in Heterogeneous Systems Using Streamtubes and Streamlines," SPE Reservoir Engineering, February 1996, 5-12.

39. Tran, T. and Journel, A.: "Automatic Generation of Corner-Point-Geometry Flow Simulation Grids from Detailed Geostatistical Descriptions," proceedings, 8th Stanford Center for Reservoir Forecasting Annual Workshop, Stanford University, May 8, 1995.

40. Verma, S., Aziz K. and Fayers J.: "Three Dimensional Voronoi Grids in Reservoir Simulation," Third SIAM Conference on Mathematical and Computational Issues in the Geosciences, San Antonio, February 8-10, 1995.

41. Verma, S.: "A Flexible Gridding Scheme for Reservoir Simulation," Proceedings, Production Operations and Engineering/General, SPE ATCE, Oct 22-25, 1995, Dallas, Texas, 657-672.

42. Verma, S.: "Flexible Grids for Reservoir Simulation," Ph.D. Dissertation, Department of Petroleum Engineering, Stanford University, June 1996.

43. Verma, S.: "The FLEX Manual," Department of Petroleum Engineering, Stanford University, June 1996. 